\title{
An evaluation of rural-urban disparities in treatment outcomes for obstructive sleep apnoea: study protocol for a prospective cohort study
}

\author{
Jennifer Corrigan ${ }^{1}$, Imhokhai Ogah ${ }^{1}$, Ada Ip-Buting $\mathbb{1}^{2}$, Heather Sharpe ${ }^{2}$, \\ Cheryl R. Laratta ${ }^{3}$, Peter Peller ${ }^{4}$, Willis H. Tsai ${ }^{1,5}$ and Sachin R. Pendharkar ${ }^{1,5}$
}

Affiliations: ${ }^{1}$ Dept of Medicine, Cumming School of Medicine, University of Calgary, Calgary, Canada. ${ }^{2}$ W21C Research and Innovation Centre, Cumming School of Medicine, University of Calgary, Calgary, Canada. ${ }^{3}$ Dept of Medicine, Faculty of Medicine and Dentistry, University of Alberta, Edmonton, Canada. ${ }^{4}$ Spatial and Numeric Data Services, University of Calgary, Calgary, Canada. ${ }^{5}$ Dept of Medicine and Community Health Sciences, Cumming School of Medicine, University of Calgary, Calgary, Canada.

Correspondence: Sachin R. Pendharkar, Cumming School of Medicine, TRW Building, Rm 3E23, University of Calgary, 3280 Hospital Drive NW, Calgary, AB, T2N 4Z6, Canada. E-mail: Sachin.pendharkarqucalgary.ca

\section{ABSTRACT}

Background: Obstructive sleep apnoea (OSA) is a common and treatable chronic condition that is associated with significant morbidity and economic cost. Geography is increasingly being recognised as a barrier to diagnosis and treatment of many chronic diseases; however, no study to date has investigated the impact of place of residence on health outcomes in OSA.

Objective: The purpose of this study is to determine whether treatment outcomes for patients initiating continuous positive airway pressure (CPAP) for OSA differ between those who live in urban versus rural settings.

Methods: A prospective cohort design will be used. Participants will be recruited through communitybased CPAP providers and assigned to either the rural or urban cohort based on residential postal code. The primary outcome will be the difference in nightly hours of CPAP use between the two groups, measured 3 months after initiation of therapy. Secondary outcomes will include symptoms, quality of life, patient satisfaction and patient-borne costs.

Anticipated results: This study will determine whether there are differences in CPAP adherence or patient-reported outcomes between rural and urban patients with OSA. These results will highlight potential challenges with providing OSA care in rural populations and may inform health interventions to reduce urban-rural inequities.

$@$ ERSpublications

Do obstructive sleep apnoea treatment outcomes differ between rural and urban populations? Engagement of community providers enables a novel approach to real-world evaluation. \#sleep @ERSpublications https://bit.ly/3cGps6X

Cite this article as: Corrigan J, Ogah I, Ip-Buting A, et al. An evaluation of rural-urban disparities in treatment outcomes for obstructive sleep apnoea: study protocol for a prospective cohort study. ERJ Open Res 2020; 6: 00141-2020 [https://doi.org/10.1183/23120541.00141-2020].

This article has supplementary material available from openres.ersjournals.com.

Received: 22 March 2020 | Accepted after revision: 03 June 2020

Copyright $\odot$ ERS 2020. This article is open access and distributed under the terms of the Creative Commons Attribution Non-Commercial Licence 4.0. 


\section{Introduction}

\section{Background and rationale}

Obstructive sleep apnoea (OSA) is a common chronic condition affecting almost 1 billion people globally, with an estimated prevalence of $17-23 \%$ in women and $34-50 \%$ in men [1-3]. Untreated OSA is associated with poor sleep, reduced quality of life, neurocognitive impairment and systemic comorbidities including metabolic, cardiovascular and cerebrovascular disease [4-8].

The estimated economic cost of untreated sleep apnoea in the United States is USD150 billion per year, attributable to disease sequelae, lost productivity and more frequent motor vehicle and workplace accidents [9]. Treatment of moderate to severe OSA with continuous positive airway pressure (CPAP) has been demonstrated to be clinically beneficial and cost-effective $[10,11]$. Despite this, achieving treatment targets such as adequate adherence to CPAP remains an ongoing challenge [12, 13].

Geography is an increasingly recognised contributor to health inequity, yet no study to date has investigated whether OSA treatment outcomes differ between rural and urban populations. Rural residence contributes to vulnerability and inferior outcomes in many chronic and treatable diseases akin to OSA. For example, individuals that live in rural or remote settings have higher rates of preventable mortality due to cardiovascular disease and cancer than their urban counterparts [14]. Rural patients with chronic diseases report the long distance from health services and low availability of healthcare professionals as barriers to treatment. An additional contributor to worse outcomes may be "rural culture", which refers to values and characteristics commonly seen in individuals that reside outside urban centres; namely self-reliance, low health literacy and reticence to seek care in urban centres due to feelings of not belonging [15].

Whether or not these factors apply to rural OSA populations is unknown. Barriers to access for diagnosis of OSA may be present, as a higher severity of OSA has been identified in patients with a longer travel time to a specialised sleep facility [16]. Historically, the need for specialist assessment and polysomnography (PSG) in order to obtain a diagnosis of OSA required rural patients to travel, often over long distances, to a sleep facility in an urban centre [17]. With the rising use of pathways incorporating home sleep apnoea tests (HSATs) and ambulatory CPAP titration [18], OSA care can now be delivered within rural communities. However, the impact of this distributed model of OSA care has not been evaluated.

\section{Study objectives}

The primary objective of this study is to determine if CPAP adherence differs between adults with uncomplicated OSA who live in rural versus urban settings. The secondary objective is to determine whether the outcomes of CPAP therapy are affected by the way that patients obtain OSA care, such as the care pathway (community versus sleep facility), distance travelled for OSA care and wait times for care.

\section{Methods}

\section{Study design}

This is a prospective cohort study designed to compare the outcomes of CPAP therapy for uncomplicated OSA between urban and rural patients. The study design is shown in figure 1.

\section{Study setting}

This study will be conducted in Alberta, Canada, where the delivery of OSA care follows one of two main pathways. Primary care physicians often represent the first point of contact and may refer individuals with suspected OSA to either a sleep specialist or a community-based respiratory homecare provider (see figure 2). Respiratory homecare providers are community-based private facilities that administer HSATs for diagnosis, dispense positive airways pressure (PAP) equipment and provide follow-up care for patients on PAP therapy. They are typically staffed by registered respiratory therapists (RRTs) who are licensed by the respiratory therapy college in the province of Alberta. The HSAT is interpreted by a board-certified/board eligible (BC/BE) sleep physician, and management decisions are deferred to the referring physician. After receiving the HSAT report, the primary care physician may choose to initiate therapy through the respiratory homecare provider or refer to a sleep specialist for further assessment and management.

The sleep specialist pathway may include an HSAT or PSG at the discretion of the sleep specialist. There are 11 accredited sleep laboratories that perform PSG in Alberta: three urban public facilities and eight private facilities in both urban and rural settings. There is no direct public funding for HSATs; these tests are typically performed in sleep laboratories or by respiratory homecare providers at no cost to the patient. The cost of fixed or auto-titrating CPAP therapy is often borne by the patient, either out-of-pocket or through private insurance, but may be covered by government assistance programmes, which often require a PSG to determine funding eligibility. Although there is regional variability in the delivery and funding of OSA care in Canada, specialty- and community-based models are available in most provinces [17, 19]. 


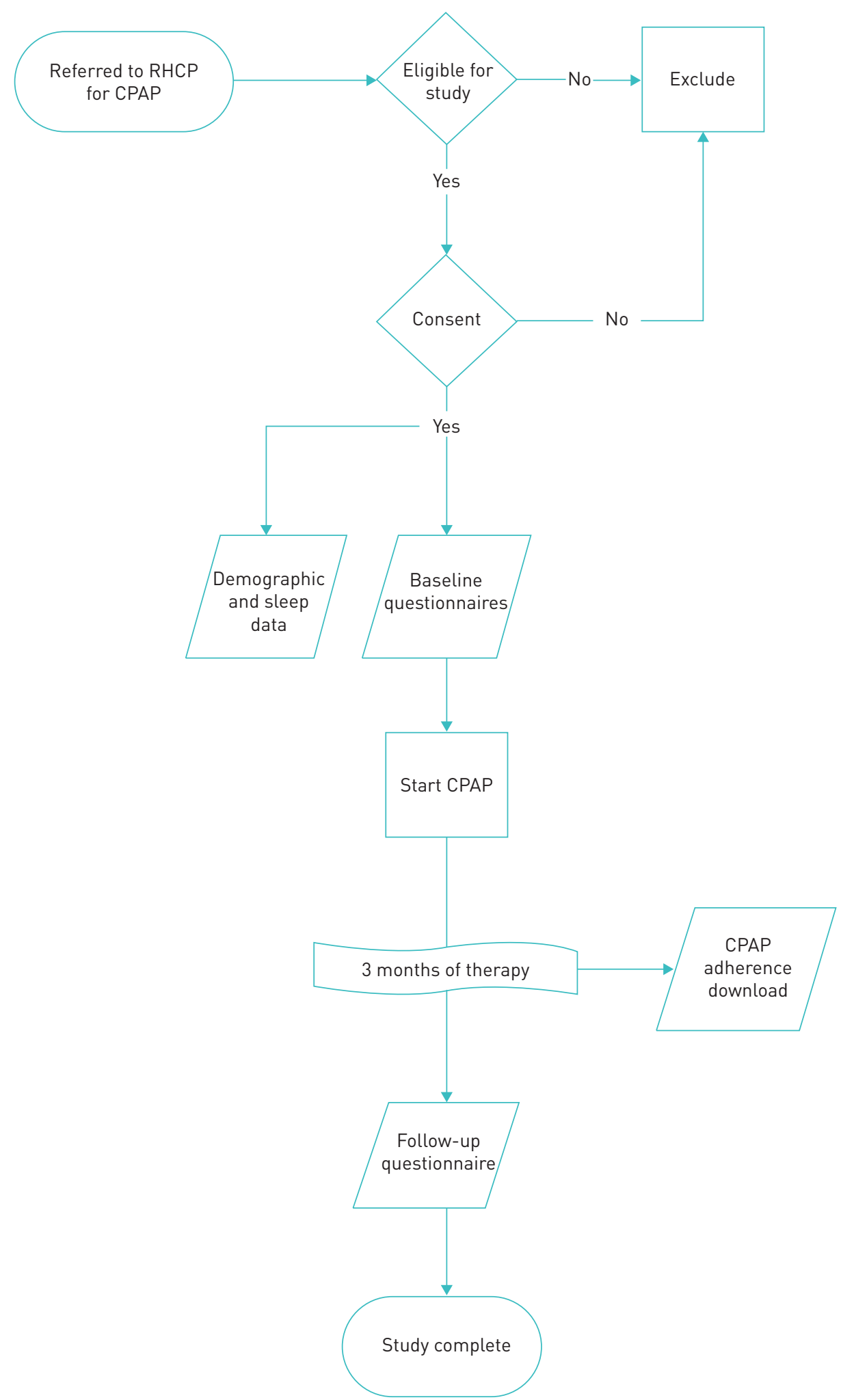

FIGURE 1 Study design flow diagram: recruitment and data collection. RHCP: respiratory homecare provider; CPAP: continuous positive airway pressure.

Eligibility criteria

Patients will be included if they meet all of the following criteria: age 18 years or older, a new diagnosis of uncomplicated OSA made by a physician using an HSAT or PSG and initiation of treatment with CPAP. 


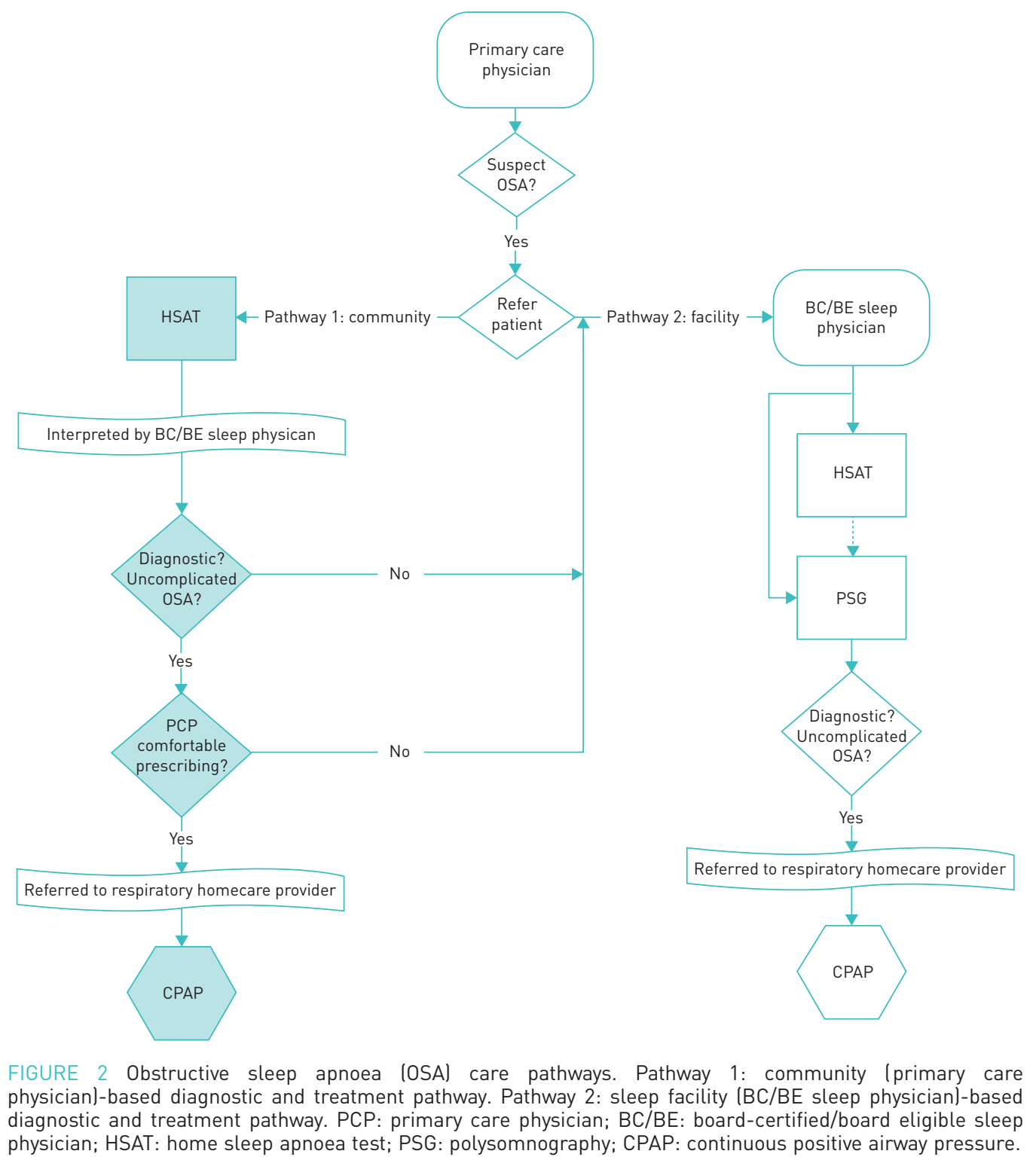

Exclusion criteria are: presence of another form of sleep-disordered breathing such as central sleep apnoea or hypoventilation, initiation of PAP therapies besides fixed or autotitrating CPAP (e.g. bilevel PAP, adaptive servoventilation), previous or current treatment with PAP therapy or oxygen, or failure to provide consent. The exclusion criteria have been designed to capture a patient population that could be safely managed in the community setting (i.e. patients with uncomplicated OSA) [20].

\section{Cohort definition}

Urban versus rural residence

Patients will be classified as rural or urban using residential postal code. The patients' postal codes will be translated into census geographic areas using the Statistics Canada Postal Code Conversion File Plus (PCCF+; Statistics Canada, ON, Canada), version 7C [21]. PCCF+ is a digital file that provides correspondence between the Canada Post Corporation six-character postal code and Statistics Canada's standard geographic areas (block face, dissemination block or dissemination area). PCCF+ can be used to classify dissemination areas based on degree of metropolitan influence (census metropolitan area type), allowing for accurate urban-rural classification. Based on Statistics Canada's definition of a large urban centre as one with a population $>100000$ [22], patients with postal codes assigned a "Community Size Classification" (CSC) of 1, 2 or 3 will be classified as urban (population >100000) and patients with a CSC of 4 or 5 will be classified as rural residents [21]. 
Community versus specialist pathway

Patients will also be classified based on the care pathway followed for OSA care, as defined in figure 2. Patients that convert from pathway 1 (community) to pathway 2 (sleep facility) will be assigned to the sleep facility pathway for analysis.

\section{Study outcomes}

The primary outcome is the mean daily hours of CPAP use recorded by the PAP device, compared between rural and urban patients. Adherence will be based on the number of hours the device has been worn in the last 4 weeks of the 3-month study period. Secondary outcomes include: the proportion of patients in each group who are adherent, defined as using CPAP for at least $4 \mathrm{~h}$ on $70 \%$ of nights [23], the change in Epworth Sleepiness Scale (ESS) total score from baseline to 3 months, change in EuroQOL (EQ-5D-3L) quality of life utility score from baseline to 3 months, Visit-Specific Satisfaction Instrument (VSQ-9) total score at 3 months and patient-reported costs at 3 months.

\section{Sample size}

Previous studies have suggested that $0.5 \mathrm{~h}$ is the minimally clinically important difference in CPAP adherence [24]. Using this adherence threshold with a 1-h standard deviation, a sample size of 200 patients (100 per group) will be required to detect a difference between rural and urban patients with $90 \%$ power and a two-sided Type I error rate of 0.025 . Given an anticipated $80 \%$ response rate to the follow-up questionnaire, a total of 250 patients will be recruited.

\section{Recruitment}

Participants will be recruited from respiratory homecare providers in Alberta at the time of initiation of CPAP therapy. Respiratory homecare providers have been invited to participate through a provincial homecare association whose member companies have agreed to meet a minimum standard of care (e.g. HSATs interpreted by $\mathrm{BC} / \mathrm{BE}$ sleep physicians, CPAP initiation and clinical follow-up by RRTs). Homecare providers involved in the study will identify potentially eligible patients, discuss the study and provide recruitment materials to individuals who are interested in participating. They will also provide study data to the research team for consenting participants. No financial incentive will be provided to homecare companies for involvement in the study.

To optimise study feasibility, meetings will be held with each participating respiratory homecare provider to develop a unique workflow process that promotes adherence to study methods while simultaneously limiting disruptions to the company's daily operations. Examples of specific considerations for each workflow process include incorporation of alerts within electronic medical records for eligible patients, a determination of whether consent forms and initial questionnaires will be administered electronically or by paper, and the method of data sharing. A designated study contact will also be identified for each homecare provider, to facilitate smooth communication between the research team, homecare employees and participants

\section{Data collection}

The timeline of data collection is outlined in table 1. Demographic data such as age, sex and postal code will be obtained directly from the respiratory homecare provider.

PCCF+ Version 7C [21] in conjunction with SAS statistical software (SAS Institute, Inc) will be used to assign patients to the urban or rural cohort as described above (see "Cohort definition"). Beyond 20/20 Professional Browser [25] will then be used to capture additional census information of the dissemination area where the patient lives, including average income per person and highest level of education. ArcMap [26] will be used to generate a road network layer for calculation of distance $(\mathrm{km})$ and time $(\mathrm{min})$ travelled to the nearest level I sleep facility.

Sleep diagnostic data will also be obtained from respiratory homecare providers. HSATs are typically performed using the ApneaLink (ResMed, San Diego, CA, USA) or the Remmers Sleep Recorder (Sagatech Electronics Inc., Calgary, Canada). Both devices are Level 3 HSAT devices that record heart rate, oxygen saturation, airflow and snoring. We will randomly select $10 \%$ of HSAT studies to validate the respiratory disturbance index (RDI) from the Remmers Sleep Recorder or respiratory event index (REI) from the ApneaLink through a review of raw data. CPAP usage data, including the number of hours worn per night (usage), mask leak and residual RDI/REI, will be acquired from machine downloads. This information is stored on a removable chip or in cloud storage depending on the CPAP device and is downloaded by the respiratory homecare provider at every visit to facilitate titration of CPAP therapy and improve adherence.

A total of four different questionnaires will be administered to each participant. These include: 


\begin{tabular}{|c|c|c|}
\hline Data collected & Baseline $^{\#}$ & Follow-up ๆ \\
\hline Demographics (age, sex, BMI, postal codel & $x$ & \\
\hline Sleep study (RDI, REI, AHI, nocturnal $S_{\mathrm{pO}_{2}}$ ) & $x$ & \\
\hline CPAP adherence (machine download) & & $x$ \\
\hline ESS & $x$ & $x$ \\
\hline VSQ-9 & & $x$ \\
\hline$E Q-5 D-3 L$ & $x$ & $x$ \\
\hline Wait times and cost of OSA care ${ }^{+}$ & $x$ & $x$ \\
\hline \multicolumn{3}{|c|}{$\begin{array}{l}\text { BMI: body mass index; RDI: respiratory disturbance index; REI: respiratory event index; } A H I: \\
\text { apnoea-hypopnoea index; nocturnal } \mathrm{S}_{\mathrm{pO}_{2} \text { : mean and lowest nocturnal oxygen saturation; CPAP: continuous }} \text { positive airway pressure; ESS: Epworth Sleepiness Scale; VSQ-9: Visit-Specific Satisfaction Instrument; } \\
\text { EQ-5D-3L: EuroQOL-5D-3 level; OSA: obstructive sleep apnoea. \#: Baseline data will be collected from } \\
\text { consenting patients at the time of CPAP initiation; ๆ: follow-up data will be collected after } 3 \text { months of } \\
\text { treatment with CPAP; }{ }^{+} \text {: baseline and follow-up "Wait Times and Cost of OSA Care" questionnaires include } \\
\text { different questions. See supplementary appendix } 3 \text {. }\end{array}$} \\
\hline
\end{tabular}

Epworth Sleepiness Scale (ESS) - The ESS is a validated patient-reported measure of daytime sleepiness and will be used to evaluate OSA symptom severity at baseline and 3 months [27]. The ESS includes eight questions assessing how likely a patient is to fall asleep in different scenarios on a scale of 0 to 3 . A higher score indicates more severe sleepiness.

Visit-Specific Satisfaction Instrument (VSQ-9) - Patient satisfaction is an important determinant of CPAP adherence [28]. The VSQ-9 is a simple, validated nine-question tool to measure patient satisfaction with an outpatient visit [29]; it has been used in previous studies evaluating care delivery models for OSA [30]. The questionnaire asks participants to rate aspects of care, such as length of time spent waiting for an appointment or the quality of the provider's explanation of their condition, using a Likert scale (see supplementary appendix 1). The VSQ-9 will be administered at 3 months.

EuroQOL 5D (EQ-5D-3L) - The EQ-5D is a standardised instrument used to measure general health-related quality of life. Quality of life scores are obtained by combining patient ranking of level of impairment in generic health areas such as mobility and mood with an overall visual analogue scale of quality of life. The EQ-5D-3L (three-level) ranking instrument will be administered at baseline and 3 months (see supplementary appendix 2).

Wait Times and Costs of OSA Care - We have developed a brief questionnaire to determine how long patients waited to receive OSA care, patient-borne costs and distance travelled to obtain care. Questions related to these aspects of care will be administered at baseline (see supplementary appendix $3 \mathrm{a}$ ) and after 3 months of therapy (see supplementary appendix $3 b$ ).

Patients will have the option of completing the questionnaires on paper or electronically via Research Electronic Data Capture (REDCap), which can be accessed securely on any smartphone or computer. QR codes will be provided to participants for easy access to the electronic link. At the time of consent, patient contact information, including telephone number, e-mail and mailing address, will be collected to facilitate follow-up. Patients will be sent an automatically generated e-mail after 3 months of therapy that provides a link to the follow-up questionnaires. Patients who do not complete follow-up questionnaires will be sent automatic e-mail reminders weekly for 4 weeks. After 4 weeks, paper questionnaires and a postage paid envelope will be mailed to participants. A study team member will contact patients via telephone 2 weeks after the paper questionnaire has been mailed to ensure it has been received.

\section{Analysis}

Descriptive statistics will be used to report baseline patient characteristics, including demographics, OSA severity and baseline questionnaire data in each study group (rural versus urban). Data will be assessed for normality, and t-tests or Mann-Whitney $U$ tests will be used for analysis of continuous variables as appropriate. Chi-squared tests will be used to compare CPAP adherence as a binary variable $(\geqslant 4 \mathrm{~h} / \mathrm{night}$ for $70 \%$ of nights) between urban and rural groups, as well as for categorical data from the Wait Times and Costs questionnaire.

Multiple linear regression analysis will be performed using hours of nightly CPAP use as the dependent variable. Independent variables will be based on the study question and other potential predictors of 
CPAP adherence from literature review and expert consensus; these include age, sex, education level, household income, OSA severity, baseline ESS, change in ESS, VSQ-9 score, urban versus rural residence, community versus facility pathway, distance to nearest sleep specialty facility, distance travelled for diagnostic testing and time to treatment. Statistical significance will be indicated by $p$-values $<0.05$. All statistical analyses will be performed using STATA (Statacorp, 2019).

\section{Ethics}

Research ethics approval

Ethics approval was obtained from the Conjoint Health Research Ethics Board (CHREB ID: REB16-2230) at the University of Calgary. The ethics approval process involved reviewing the study with respect to content and compliance with applicable research and safety regulations. In addition to the initial approval of the study, the CHREB will review the study on an annual basis.

\section{Protocol amendments}

Any modification to the study protocol will require a formal amendment to the protocol and submission to the CHREB for approval. Modifications will be communicated to relevant stakeholders including homecare providers and study participants.

\section{Consent or assent}

Study participants will complete an informed consent form prior to initial questionnaires. A member of the respiratory homecare provider team will inform eligible patients of the study and provide access to an electronic or paper consent form. The form outlines the study purpose and details, including risks and benefits, at a grade seven reading level. Readability was verified using the Flesch-Kincaid Tool [31].

Questions regarding the study will be directed to the study research associate either by phone or e-mail. The electronic version employs a "click-through" method, in which participants must first read the consent form and then click that he/she understands and agrees to participate, in lieu of a signature. Similarly, the paper consent form must be signed prior to completing the paper questionnaires. Participants can withdraw from the study at any time, either completely or for questionnaires only. Data contributed up to the point of withdrawal will be retained and if a participant withdraws from questionnaires only, we will collect CPAP download data at 3 months.

\section{Data storage and confidentiality}

In accordance with research ethics board approval, questionnaire data will be stored on a secure network within University of Calgary firewalls and will be accessed by the investigators and research associates using password-protected entry to the questionnaire database (Research Data Capture; REDCap). Study questionnaires will be administered using REDCap via a secure electronic link, or on paper if preferred by patients. Paper responses will be entered into REDCap by a research assistant. CPAP adherence downloads and other patient data will be stored securely and in accordance with University of Calgary Research Ethics Board requirements.

\section{Anticipated results, dissemination and knowledge translation}

With almost one billion people affected worldwide [3], untreated OSA imparts a large global health, financial and safety burden. While this burden can be mitigated with treatment of OSA, there are several potential barriers that may hinder effective treatment. Geography is one such barrier that has been shown to lead to variation in the treatment of chronic diseases but has not been investigated for OSA. Studies to date have shown that individuals who travel farther to reach a sleep facility are more likely to report symptoms of OSA despite not having a formal diagnosis [32], and have a higher apnoea-hypopnoea index when they are diagnosed [16]. Despite this disparity based on distance from a sleep facility, no study has evaluated whether geography affects OSA treatment outcomes.

This study will address multiple topics identified as high priority in OSA research, such as the identification of barriers and facilitators of effective treatment in various geographic populations [33, 34]. The engagement of community respiratory homecare providers will enable recruitment of a geographically diverse population of OSA patients, generating real-world data on possible urban-rural differences that will be generalisable to the sleep community at large.

The use of Statistics Canada PCCF+ [21] and Beyond 20/20 Professional Browser [25] enables an innovative approach to evaluating health impacts of poor access to specialist care in rural areas. Previous studies have used travel distance or time to sleep specialist care as a crude measure of "rurality". Postal code-linked census data afford us the ability to accurately assign patients to a rural or urban cohort using population size and metropolitan influence. Furthermore, for the first time, the effect of possible 
confounding variables such as patient income, education level and out-of-pocket cost will be assessed. Ascertaining this information is the first step in developing effective, contextually relevant and patient-centred models of care [35-37].

The results of this study will be presented at academic conferences and published in peer-reviewed journals, as well as shared with local providers and policymakers. Through this broad dissemination approach, we will share novel findings about the impact of rural residence on treatment outcomes for OSA. More broadly, this study will provide additional information about the challenges of delivering high-quality care to patients from geographically dispersed communities.

Author contributions: J. Corrigan conceived and designed the study, analysed and interpreted the data, and drafted and made critical revisions to the manuscript; I. Ogah conceived and designed the study, and drafted and made critical revisions to the manuscript; A. Ip-Buting acquired data and made critical revisions to the manuscript; $\mathrm{H}$. Sharpe and C.R. Laratta made critical revisions to the manuscript; P. Peller acquired data (PCCF+, SAS, Beyond 2020) and made critical revisions to the manuscript; W.H. Tsai and S.R. Pendharkar conceived and designed the study, analysed and interpreted the data, and drafted and made critical revisions to the manuscript.

Conflict of interest: J. Corrigan has nothing to disclose. I. Ogah has nothing to disclose. A. Ip-Buting reports grants from The Lung Association and the Respiratory Health Strategic Clinical Network during the conduct of the study. H. Sharpe has nothing to disclose. C.R. Laratta reports personal fees from the RANA Respiratory Care Group for the renumeration of home sleep apnoea tests outside the submitted work. P. Peller has nothing to disclose. W.H. Tsai has nothing to disclose. S.R. Pendharkar has nothing to disclose.

Support statement: This study was supported by The Lung Association, Alberta and Northwest Territories National Grant Review/Grant-In-Aid Program, an Innovation and Seed Grant awarded by Alberta Health Services' Respiratory Health Strategic Clinical Network (RHSCN), and by an RHSCN summer studentship. Funding agencies had no role in study design, analysis or interpretation of results. Funding information for this article has been deposited with the Crossref Funder Registry.

\section{References}

1 Heinzer R, Vat S, Marques-Vidal P, et al. Prevalence of sleep-disordered breathing in the general population: the HypnoLaus study. Lancet Respir Med 2015; 3: 310-318.

2 Peppard PE, Young T, Barnet JH, et al. Increased prevalence of sleep-disordered breathing in adults. Am J Epidemiol 2013; 177: 1006-1014.

3 Benjafield AV, Ayas NT, Eastwood PR, et al. Estimation of the global prevalence and burden of obstructive sleep apnoea: a literature-based analysis. Lancet Respir Med 2019; 7: 687-698.

4 Bonsignore MR, Baiamonte P, Mazzuca E, et al. Obstructive sleep apnea and comorbidities: a dangerous liaison. Multidiscip Respir Med 2019; 14: 8.

5 Bubu OM, Pirraglia E, Andrade AG, et al. Obstructive sleep apnea and longitudinal Alzheimer's disease biomarker changes. Sleep 2019; 42: zsz048.

6 Olaithe M, Bucks RS, Hillman DR, et al. Cognitive deficits in obstructive sleep apnea: insights from a meta-review and comparison with deficits observed in COPD, insomnia, and sleep deprivation. Sleep Med Rev 2018; 38: 39-49.

7 Wang G, Goebel JR, Li C, et al. Therapeutic effects of CPAP on cognitive impairments associated with OSA. J Neurol; 2020; 267: 2823-2828.

8 Patel N, Donahue C, Shenoy A, et al. Obstructive sleep apnea and arrhythmia: a systemic review. Int J Cardiol 2017; 228: 967-970.

9 Watson NF. Health care savings: the economic value of diagnostic and therapeutic care for obstructive sleep apnea. J Clin Sleep Med 2016; 12: 1075-1077.

10 Tran K, Kim J, Tsoi B, et al. CADTH Optimal Use Reports. In Interventions for the Treatment of Obstructive Sleep Apnea in Adults: A Health Technology Assessment - Project Protocol. (2016).

11 Streatfeild J, Hillman D, Adams R, et al. Cost-effectiveness of continuous positive airway pressure therapy for obstructive sleep apnea: health care system and societal perspectives. Sleep 2019; 42: zsz181.

12 Bakker JP, Weaver TE, Parthasarathy S, et al. Adherence to CPAP: what should we be aiming for, and how can we get there? Chest 2019; 155: 1272-1287.

13 Rotenberg BW, Murariu D, Pang KP. Trends in CPAP adherence over twenty years of data collection: a flattened curve. J Otolaryngol Head Neck Surg 2016; 45: 43.

14 Subedi R, Greenberg TL, Roshanafshar S. Does geography matter in mortality? An analysis of potentially avoidable mortality by remoteness index in Canada. Health Rep 2019; 30: 3-15.

15 Brundisini F, Giacomini M, DeJean D, et al. Chronic disease patients' experiences with accessing health care in rural and remote areas: a systematic review and qualitative meta-synthesis. Ont Health Technol Assess Ser 2013; 13: $1-33$.

16 Allen AJMH, Amram O, Tavakoli $\mathrm{H}$, et al. Relationship between travel time from home to a regional sleep apnea clinic in British Columbia, Canada, and the severity of obstructive sleep. Ann Am Thorac Soc 2016; 13: 719-723.

17 Pendharkar SR, Povitz M, Bansback N, et al. Testing and treatment for obstructive sleep apnea in Canada: funding models must change. CMAJ 2017; 189: E1524-E1528.

18 Rosenberg R, Hirshkowitz M, Rapoport DM, et al. The role of home sleep testing for evaluation of patients with excessive daytime sleepiness: focus on obstructive sleep apnea and narcolepsy. Sleep Med 2019; 56: 80-89.

19 Fenton M, Ayas N, Povitz M, et al. Does clinical care for obstructive sleep apnea differ between Canadian jurisdictions with and without government funding for CPAP? Results of an online patient survey. Sleep Med 2019; 64: S111-S112.

20 Rosen IM, Kirsch DB, Carden KA, et al. Clinical use of a home sleep apnea test: an updated American Academy of Sleep Medicine Position Statement. J Clin Sleep Med 2018; 14: 2075-2077. 
Statistics Canada. Postal Code Conversion File Plus (PCCF+) Version 7C. (2019).

Statistics Canada. Population Centre and Rural Area Classification 2016 - Definitions. (2016).

Kribbs NB, Pack AI, Kline LR, et al. Objective measurement of patterns of nasal CPAP use by patients with obstructive sleep apnea. Am Rev Respir Dis 1993; 147: 887-895.

Patil SP, Ayappa IA, Caples SM, et al. Treatment of adult obstructive sleep apnea with positive airway pressure: an American Academy of Sleep Medicine systematic review, meta-analysis, and GRADE assessment. J Clin Sleep Med 2019; 15: 301-334.

Statistics Canada. Beyond 20/20 Professional Browser. (2014).

Environmental Systems Research Institute. ArcMap. (2019).

Johns MW. A new method for measuring daytime sleepiness: the Epworth Sleepiness Scale. Sleep 1991; 14 540-545.

Sawyer AM, Gooneratne NS, Marcus CL, et al. A systematic review of CPAP adherence across age groups: clinical and empiric insights for developing CPAP adherence interventions. Sleep Med Rev 2011; 15: 343-356.

Rubin HR, Gandek B, Rogers WH, et al. Patients' ratings of outpatient visits in different practice settings. Results from the Medical Outcomes Study. JAMA 1993; 270: 835-840.

Pendharkar SR, Tsai WH, Penz ED, et al. A randomized controlled trial of an alternative care provider clinic for severe sleep-disordered breathing. Ann Am Thorac Soc 2019; 16: 1558-1566.

Yeung AWK, Goto TK, Leung WK. Readability of the 100 most-cited neuroimaging papers assessed by common readability formulae. Front Hum Neurosci 2018; 12: 308.

Spagnuolo CM, McIsaac M, Dosman J, et al. Distance to specialist medical care and diagnosis of obstructive sleep apnea in rural Saskatchewan. Can Respir J 2019; 2019: 1683124.

Patel K, Moorthy D, Chan JA, et al. High priority future research needs for obstructive sleep apnea diagnosis and treatment. J Clin Sleep Med 2013; 9: 395-402.

34 George M, Hernandez C, Smith S, et al. Nursing research priorities in critical care, pulmonary, and sleep: International Delphi survey of nurses, patients, and caregivers - an official American Thoracic Society workshop report. Ann Am Thorac Soc 2020; 17: 1-10.

5 Roorda C, de Bock GH, Scholing C, et al. Patients' preferences for post-treatment breast cancer follow-up in primary care vs. secondary care: a qualitative study. Health Expect 2015; 18: 2192-2201.

Jackson GL, Powers BJ, Chatterjee R, et al. The patient centered medical home. A systematic review. Ann Intern Med 2013; 158: 169-178.

7 Grumbach K, Selby JV, Damberg C, et al. Resolving the gatekeeper conundrum: what patients value in primary care and referrals to specialists. JAMA 1999; 282: 261-266. 